\title{
Erratum to: A Wald test with enhanced selectivity properties in homogeneous environments
}

Weijian Liu ${ }^{1,2^{*}}$, Wenchong $\mathrm{Xie}^{2}$ and Yongliang Wang ${ }^{2}$

After publication of our work [1] we noticed the following mistakes:

Equation (3) should be

$$
\boldsymbol{I}(\boldsymbol{\theta})=\mathrm{E}\left[\left(\frac{\partial \ln f(\boldsymbol{x}, \boldsymbol{X} ; \boldsymbol{\theta})}{\partial \boldsymbol{\theta}^{*}}\right)\left(\frac{\partial \ln f(\boldsymbol{x}, \boldsymbol{X} ; \boldsymbol{\theta})}{\partial \boldsymbol{\theta}^{T}}\right)\right]
$$

Equation (5) should be

$$
t_{\mathrm{Wald}}=\left(\hat{\boldsymbol{\theta}}_{r_{1}}-\boldsymbol{\theta}_{r_{0}}\right)^{H}\left\{\left[\boldsymbol{I}^{-1}\left(\hat{\boldsymbol{\theta}}_{1}\right)\right]_{\boldsymbol{\theta}_{\boldsymbol{\theta}_{r}} \boldsymbol{\theta}_{r}}\right\}^{-1}\left(\hat{\boldsymbol{\theta}}_{r_{1}}-\boldsymbol{\theta}_{r_{0}}\right)
$$

The notation "-" in equations (6), (11), and (12) should be replaced by "-“, i.e., the minus sign.

Equations (17), (18), and (19) should be

$$
\begin{aligned}
\tilde{t}_{\mathrm{ACE}} & =\frac{\tilde{\boldsymbol{x}}^{H} \boldsymbol{P} \tilde{\boldsymbol{s}} \tilde{\boldsymbol{x}}}{\tilde{\boldsymbol{x}}^{H} \boldsymbol{P} \tilde{\boldsymbol{s}}^{\perp} \tilde{\boldsymbol{x}}}=\frac{\|\boldsymbol{P} \tilde{\boldsymbol{s}} \tilde{\boldsymbol{x}}\|^{2}}{\left\|\boldsymbol{P} \tilde{\boldsymbol{s}}^{\perp} \tilde{\boldsymbol{x}}\right\|^{2}} \\
t_{\text {Wald }} & =\frac{1}{1+\|\tilde{\boldsymbol{x}}\|^{2}\left\|\boldsymbol{P} \tilde{\boldsymbol{s}}^{+} \tilde{\boldsymbol{x}}\right\|^{2} /\|\boldsymbol{P} \tilde{\boldsymbol{s}} \tilde{\boldsymbol{x}}\|^{2}}
\end{aligned}
$$

and

$$
\tilde{t}_{\mathrm{Wald}}=\frac{\|\boldsymbol{P} \tilde{\boldsymbol{s}} \tilde{\boldsymbol{x}}\|^{2}}{\left\|\boldsymbol{P} \tilde{\boldsymbol{s}}^{\perp} \tilde{\boldsymbol{x}}\right\|^{2}\|\tilde{\boldsymbol{x}}\|^{2}}
$$

respectively.

Published online: 24 July 2015

\section{References}

1. W Liu, W Xie, Y Wang, A Wald test with enhanced selectivity properties in homogeneous environments. EURASIP J Adv Signal Process 2013, 14 (2013)

\footnotetext{
*Correspondence: liuvjian@163.com

${ }^{1}$ College of Electronic Science and Engineering, National University of Defense Technology, 410073, Changsha, China

${ }^{2}$ Key Research Lab, Wuhan Radar Academy, 430019 Wuhan, China
}

Submit your manuscript to a SpringerOpen ${ }^{\circ}$ journal and benefit from:

- Convenient online submission

- Rigorous peer review

- Immediate publication on acceptance

- Open access: articles freely available online

- High visibility within the field

- Retaining the copyright to your article

Submit your next manuscript at $>$ springeropen.com

\section{黑 Springer}

(c) 2015 Liu et al. This is an Open Access article distributed under the terms of the Creative Commons Attribution License (http://creativecommons.org/licenses/by/4.0), which permits unrestricted use, distribution, and reproduction in any medium, provided the original work is properly credited. 